\title{
EFFICIENT ENERgY UTILIZATION PATH Algorithm IN Wireless SENSOR NETWORKS
}

\author{
K.Ramanan ${ }^{1}$ and E.Baburaj ${ }^{2}$ \\ ${ }^{1}$ Sathyabama University /Department of Computer Science\& Engineering, Chennai, \\ India \\ ${ }^{2}$ Narayanaguru College of Engineering\&Technology /Department of Computer \\ Science\& Engineering, Nagercoil, India
}

\begin{abstract}
With limited amount of energy, nodes are powered by batteries in wireless networks. Increasing the life span of the network and reducing the usage of energy are two severe problems in Wireless Sensor Networks. A small number of energy utilization path algorithms like minimum spanning tree reduces total energy consumption of a Wireless Sensor Network, however very heavy load of sending data packets on many key nodes is used with the intention that the nodes quickly consumes battery energy, by raising the life span of the network reduced. Our proposal work aimed on presenting an Energy Conserved Fast and Secure Data Aggregation Scheme for WSN in time and security logic occurrence data collection application. To begin with, initially the goal is made on energy preservation of sensed data gathering from event identified sensor nodes to destination. Invention is finished on Energy Efficient Utilization Path Algorithm (EEUPA), to extend the lifespan by processing the collecting series with path mediators depending on gene characteristics sequencing of node energy drain rate, energy consumption rate, and message overhead together with extended network life span. Additionally, a mathematical programming technique is designed to improve the lifespan of the network. Simulation experiments carried out among different relating conditions of wireless sensor network by different path algorithms to analyze the efficiency and effectiveness of planned Efficient Energy Utilization Path Algorithm in wireless sensor network (EEUPA).
\end{abstract}

\section{KEYWORDS}

Wireless Sensor Network (WSN), Environmental Monitoring, Efficient Energy Utilization Path Algorithm (EEUPA), Data Collector, Sensor Nodes.

\section{INTRODUCTION}

Nodes consists of wireless crossing point to transfer the information to each other are broadly used in Wireless Sensor Networks (WSNs) in various areas such as examination, disaster liberation, intellectual carrying, surveillance, environmental managing, healthcare, goal tracking, and more. To collect the information and data in unkind or defensive atmosphere, Wireless Sensor Networks are more useful. In a Wireless Sensor Networks, data collected by sensor nodes are preferred to be circulated to destinations (base stations). May be, data kept in few nodes might not be explicitly transmitted to the destination because the destination is far away from the radio transceivers of the wireless crossing point of the nodes. So, path protocols are preferred, where the data packets are transmitted through multi-hop manner, i.e., they are transmitted node next to node, consequently reaches the destination. 
International Journal on Cybernetics \& Informatics (IJCI) Vol. 4, No. 2, April 2015

Generally, nodes in a Wireless Sensor Networks are powered by means of batteries with limited amount of energy. As a result, network separation may occur as one or more nodes employ available energy of batteries, to supply conservative path procedure undesirable. Consequently, it is severe for a Wireless Sensor Networks to start effectively to prepare an efficient energy utilization path algorithm where the battery energy is drained out fully since the wireless sensor network has an extended lifetime. Path protocol designed at power-saving and increasing network lifespan are seriously taken in research community.

A wireless sensor network (WSN) physically consists of large amount of miniature, multifunctional and resource controlled sensors which are self-organized as an informal network to examine the physical world. Sensor networks are often used in applications where it is complicated or impossible to gather wired networks. Examples include wildlife habitat checking, security and military observation, and target path.

For applications same as military surveillance, opponents have strong motivation to hear on network transfer to achieve significant intellect. Utilization of such information can source monetary fatalities or risk human lives. To secure such information, researchers in sensor network security comprises of intended significant challenge on identifying techniques to offer characteristic security services such as privacy, substantiation, reliability, and accessibility. But, these are dangerous security provisions because they are inadequate in various reasons. The communication samples of sensors can themselves represents a huge transaction of essential information, which discloses the position information of strict components in a sensor network.

Path in wireless sensor network is very important, mainly in intolerant surroundings. Failure to protect such information can completely challenge the planned principles of sensor network applications. Path actions need to be developed to prevent the opponent from determining the physical location of source sensors and destination. Because of the limited energy life span of battery-powered sensor nodes and techniques comprises to be energy efficient. Because communication in sensor networks is more special than calculation, so we use communication cost to verify the energy consumption of the routing protocols.

To decrease the utilization of energy in the wireless sensor networks, an efficient energy utilization path algorithm in an improved version is initiated by processing the grouping series with path mediators. Path series on the particular time is taken with showing alternative form of gene character. Other hidden alternative form of gene traits is preserved for future sequencing instance on definite threshold.

\section{LITERATURE REVIEW}

The field of wireless sensor networks (WSN) is now developed in the research community area because of its applications in different fields for instance defense security, civilian applications and medical research. These limitations eliminate the utilization of traditional path protocols planned for other ad hoc wireless networks. Secondly, the base station cannot verify data reliability and accuracy via fixing message digests or signatures to every sensing model. To come across the above two disadvantages, the base station [1]. can regain all sensing data yet these data has been combined. The multi level data aggregation method are presented [6]. to develop the data collecting path for a mobile destination as Infrastructure based Data Gathering Protocol (IDGP) and a Distributed Data Gathering Protocol (DDGP). A k-hop relay mechanism is established to restrict the number of hops for path data to a mobile sink.

The key point of EEGA scheme [2]. is that accurate data aggregation is attained without discharging secret sensor readings and without initiating important overhead on the battery- 
International Journal on Cybernetics \& Informatics (IJCI) Vol. 4, No. 2, April 2015

limited sensors. To come across the delay planned an Efficient Data Collection Aware of SpatioTemporal Correlation (EAST) that utilizes shortest routes used for forwarding the

collected data toward the sink node [3]. and fully expand both spatial and temporal correlations to execute near real-time data collection in WSN. Developed [4]. easy, least-time, energy-efficient path protocol by means of one-level data aggregation which guarantees improved life span for the network. The energy-efficient data development issue [5]. through person packet delay limitations to an energy-efficient service curve construction issue is developed and solves the difficulty by developing a local optimality theorem.

To design a method to mitigate the uneven energy dissipation problem by controlling the mobility of agents, which is achieved by an energy prediction strategy to find their positions using [7]. energy balancing cluster routing based on a mobile agent (EBMA) for WSNs. To obtain this while maintaining a good trade-off between the communication overhead of the scheme, the storage space requirements on the nodes, and the ratio between the number of visited nodes $\mathrm{x}$ and the representativeness of the gathered data using [8].density-based proactive data dissEmination Protocol (DEEP), which combines a probabilistic flooding with a probabilistic storing scheme.The QoS of an energy-efficient cluster-based routing protocol called Energy-Aware routing Protocol (EAP)[9]. in terms of lifetime, delay, loss percentage, and throughput, and proposes some modifications on it to enhance its performance.

QoS of an energy-efficient cluster-based path protocol called Energy-Aware routing Protocol (EAP) in terms of lifespan, delay, loss percentage, and throughput, and presents some alterations on it to improve its results [10]. DHAC [11]. can include both quantitative and qualitative information types in clustering. It prevents the destination to collect a representative investigation of the network's sensed data. Presented efficient and balanced cluster-based data aggregation algorithm (EEBCDA) splits the network into rectangular grids [12]. with uneven size and creates cluster heads rotate between the nodes in every grid correspondingly, the grid whose cluster head utilizes more energy by means of offering unbalanced energy dissipation.

To produce system-level behaviors that show life-long adaptivity to changes and perturbations in an external environment using [13].bee-inspired BeeSensor protocol that is energy-aware, scalable and efficient. En Energy Efficient and QoS aware multipath routing protocol ( EQSR)[14]. that maximizes the network lifetime through balancing energy consumption across multiple nodes, uses the concept of service differentiation to allow delay sensitive traffic to reach the sink node within an acceptable delay, reduces the end to end delay through spreading out the traffic across multiple paths, and increases the throughput through introducing data redundancy. In this approach[15]. shares intermediate results among queries to reduce the number of messages. When the sink receives multiple queries, it should be propagated these queries to a wireless sensor network via existing routing protocols. The sink could obtain the corresponding topology of queries and views each query as a query tree. With a set of query trees collected at the sink.

The above planned research gap, encouraged us to plan a Fast and Secure Energy Efficient Data Aggregation Scheme for Wireless Sensor Network matched to applications.

\section{Methodology}

WSN is a wireless sensor network relating to the distributed device by means of sensors to observe the environmental conditions at different positions. A WSN is developed by planned sensor nodes in an application area. The Sensor Node is a very important factor of WSN, is prepared with Computation, sensing and wireless Communication unit. Wireless Sensor 
International Journal on Cybernetics \& Informatics (IJCI) Vol. 4, No. 2, April 2015

Networks are created properly for security, environmental monitoring, computerization, habitat groping and creative industries etc. A block diagram of wireless sensor network system architecture is shown in Fig.1.

A characteristic node in a WSN is generally consists of four important units:

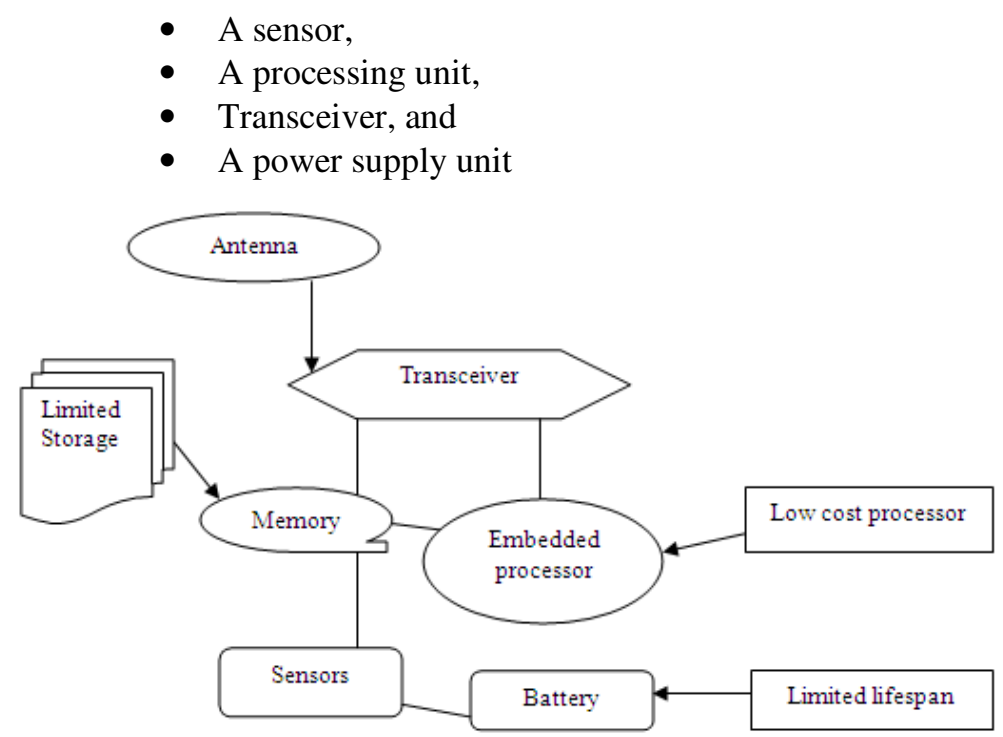

Figure 1. Block Diagram of Sensor Node

Energy utilization of a node is mainly developed as radio communications. The energy costs of broadcasting and getting a k-bit data packet between two nodes being $\mathrm{d}$ meters separately can be, equally, expressed as

$$
E_{T x}(k, d)=k\left(E_{\text {elec }}+\varepsilon_{\text {amp }} d\right)
$$

and

$$
E_{R x}(k)=k E_{\text {elec }}
$$

$\mathrm{E}_{\text {elec }}$ denotes the energy utilization due to digital coding, inflection, filtering, and diffusion of the signal, etc and $\mathrm{e}_{\mathrm{amp}}$ is the energy utilized by the source power amplifier. The energy efficiency of WSN is shown in Fig.2 


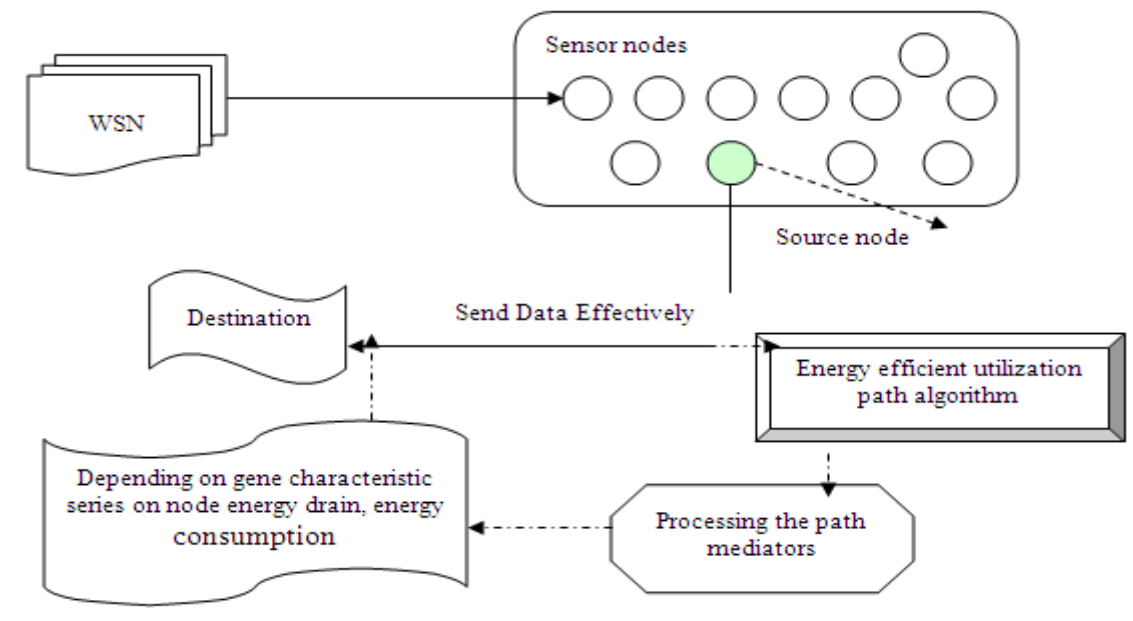

Figure 2. Architecture diagram of the proposed EEUPA

The above figure (figure 2) explains the complete process of the EEUPA system. The EEUPA is employed here for sending the sensed set of data from event identified sensor nodes to destination. For sending out the data, efficient energy utilization path algorithm is designed. The efficient energy utilization path algorithm is included by gathering the sequences of data depending on gene characteristics sequencing to improve the life span of the network. The gene characteristic series are estimated depending on the node energy drain rate and the utilization of energy required for sending out the data.

\subsection{Efficient Energy Utilization Path Algorithm (EEUPA)}

In the designed EEUPA, energy utilization of sensed data collection in the network environment is carried out by means of minimum energy utilization path algorithm. Through minimum node energy drain rate, and minimized message overhead, gene contains two properties,

- Hidden property and

- Exposed property

On processor node, drain rate series is sustained in gene property called allele. Path series at particular occurrence is taken with exposed allele of gene traits. Secondly, hidden allele of gene traits are protected for future sequencing occurrence on exact threshold. Among the frequency sets, we recognize the active path depending on the arrangement of allele in each occurrence of the nodes in the network. Consider a set of sensors planned in a field. The EEUPA system offers the following properties of the Wireless Sensor Networks:

(1) There are only one destination and sensor nodes in the WSN.

(2) The destination is inactive and the topology of the WSN collects data generated by the nodes.

(3) The destination has sufficient power supply while nodes are powered by batteries with restricted energy.

(4) Nodes do not move after they are planned, to the destination.

(5) All nodes have similar traits

The path node mediators in the network are selected depending on the gene characteristics series on energy utilization and node energy drain rate. The energy drain rate of the gene character series are recognized depending on the staying energy and the draining speed of the nodes in the 
network. The node energy drain rate is calculated depending on the genes (nodes) staying energy and the time. The energy drain rate is planned as,

$$
D R(\text { genes })=\frac{E_{P E}-E_{C E}}{T_{C T}-T_{P T}}
$$

Where,

DR (genes) - Drain rate of the gene sequences

$\mathrm{E}_{\mathrm{PE}}$ - Prior assigned energy of the particular gene

$\mathrm{E}_{\mathrm{CE}}-$ Present energy of the particular gene

$\mathrm{T}_{\mathrm{CT}}-$ Present time of the gene presence in the network

$\mathrm{T}_{\mathrm{PT}}-$ Prior time of the gene

In the WSNs, recognize the path node mediators from source to sink. After that for every occurrence of node, the utilization of energy and the node energy drain rate is calculated. To offer the effective path, choose the series of gene characteristics which has less energy drain rate and energy utilization. By tracking the system of choosing the path node mediators, the data packet is effectively transferred from source to sink in less amount of time. The algorithm below explains the process of choosing the effective path node mediators.

From Fig.3 primarily packets are sent through the path to reach the destination from source after selecting the $\mathrm{S}$ and $\mathrm{D}$ from the sensor networks. The staying energy of all the path series of node mediators in the network is subsequently calculated. The staying energy of all nodes for

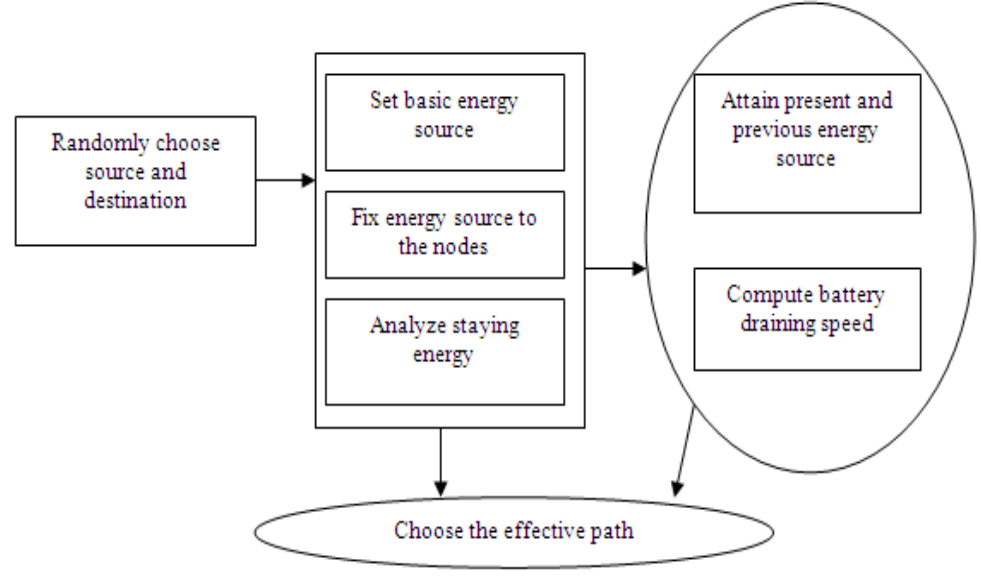

Figure 3. Processing the Path Node Mediators

particular period of time is accumulated in the file. This is used to calculate the energy draining speed. The staying energy of nodes and node energy drain rate is calculated by means of the threshold values. Compare the energy utilization of node and the energy drain with their equivalent threshold values to find out the best path. Depending on the calculated value, choose the node which has minimum value of energy utilization and drain rate of energy.

The key task of the efficient energy utilization path algorithm is to choose the maximum network lifespan for a given wireless sensor networks. Consequently, by investigating the properties of gene, the frequency set is recognized. By means of this set, the path for the particular occurrence is recognized and leads it. In the proposed EEUPA work, the path series characteristics are 
International Journal on Cybernetics \& Informatics (IJCI) Vol. 4, No. 2, April 2015

accumulated at every exposed allele and the hidden alleles are preserved for future set of path occurrences.

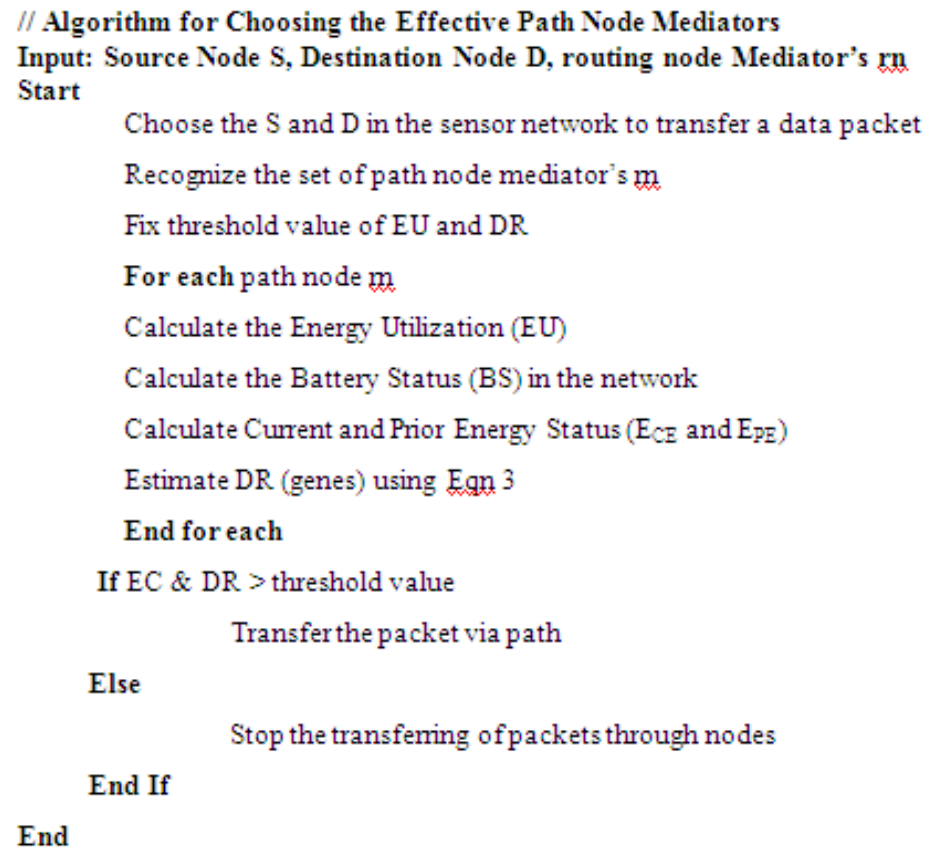

Figure 4. Process of Choosing Effective Path Node Mediators

\section{EXPERIMENTAL DisCUSSIONS}

We execute experiments to verify the proposed EEUPA system and evaluate its results. In the simulations, we used $1 / 5$ of every data as preparation period, and permit the nodes to create their primary contact history. After the preparation time, we created 1000 messages, all starting from an arbitrary source node to an arbitrary destination node for every t seconds. The period of the experiment is set as $t=300 \mathrm{~s}$. All messages are consigned a Time-To-Live (TTL) value representing the majority delay restriction.

For the discussions, we have used three set of parameters to calculate the performance of the proposed EEUPA system and compared with the existing works like LEACH (Low Energy Adaptive Cluster Hierarchy), PEGASIS (Power-Efficient Gathering in Sensor Information Systems). The Performance of EEUPA is measured in terms are listed as,

i) Node Energy Drain Rate,

ii) Message Overhead and

iii) Network Lifespan

\section{RESUlts AND DiscuSSION}

In this section, we evaluate the attained results with the existing algorithm to calculate the result of the proposed EEUPA system. The below table and graph explains the performance estimation of both the existing and proposed methods. 
International Journal on Cybernetics \& Informatics (IJCI) Vol. 4, No. 2, April 2015

\subsection{Node Energy Drain Rate}

Table 1. No. Of Nodes Vs. Node Energy Drain Rate

\begin{tabular}{|c|c|c|c|}
\hline \multirow{2}{*}{ No. of Nodes } & \multicolumn{3}{|c|}{ Node Energy Drain Rate (\%) } \\
\cline { 2 - 4 } & EEUPA Method & LEACH Scheme & PEGASIS \\
\hline 25 & 5 & 10 & 7 \\
\hline 50 & 9 & 15 & 12 \\
\hline 75 & 15 & 21 & 18 \\
\hline 100 & 19 & 28 & 25 \\
\hline 125 & 24 & 35 & 30 \\
\hline 150 & 28 & 40 & 35 \\
\hline 175 & 30 & 47 & 42 \\
\hline 200 & 32 & 58 & 49 \\
\hline
\end{tabular}

The node energy drain rate is calculated based on the number of active nodes in the wireless sensor networks and the values of the proposed Efficient Energy Utilization Path Algorithm is compared with the existing LEACH and PEGASIS schemes and illustrated in Table 1.

Fig.5 explains the drain rate as a method to report for the rate at which energy gets degenerated at a specified node. Every node observes its energy and preserves its battery power drain rate value by taking mean of the amount of energy utilization and calculating the energy dissipation per second. Compared to existing works like LEACH and heuristic model, the proposed EEUPA has small energy drain rate since it used large amount of energy for sending the data between the set of nodes in WSN. However in the proposed work, decrease the surplus dissipation of particular nodes by considering the present traffic situation and by using the drain rate of the staying battery capability.

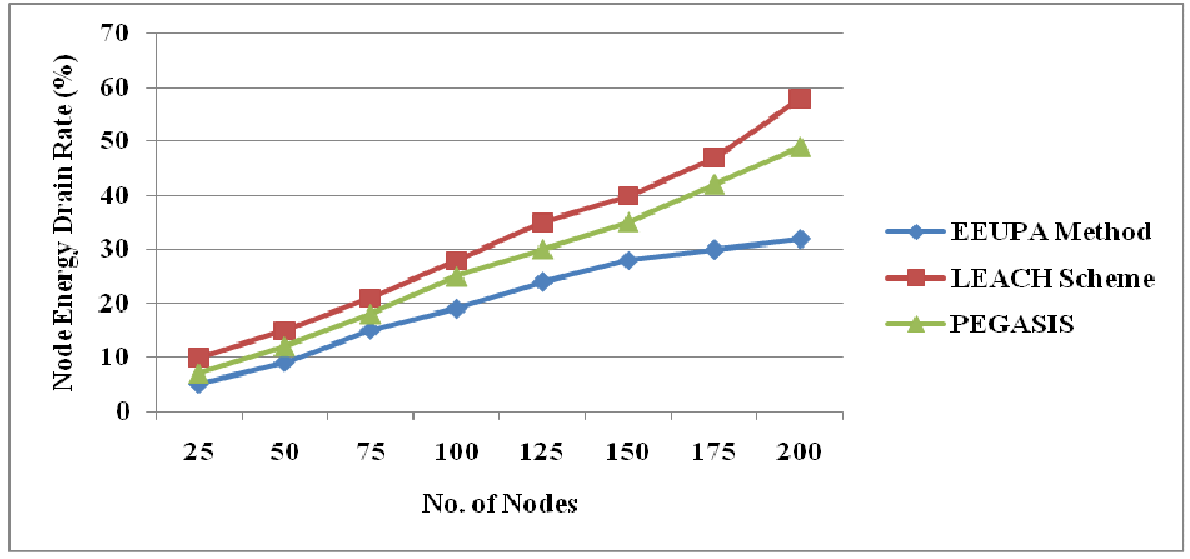

Figure 5. No. of Nodes vs. Node Energy Drain Rate

\subsection{Message Overhead}

The occurrence of message overhead is calculated depending on the number of messages to be sent into the wireless sensor networks and the values of the proposed EEUPA is compared with the existing LEACH and PEGASIS schemes is illustrated in Table 2. 
International Journal on Cybernetics \& Informatics (IJCI) Vol. 4, No. 2, April 2015

Fig.6 illustrates the existence of message overhead depending on the number of messages to be sent into the network. The message overhead is termed as the number of unsuccessful messages acquired while sending the sensed data from source to destination. Because the path verifying is completed in the proposed EEUPA, results in minimizing the message overhead on the path series for the data collection of sensed events by the destination. By comparing to the other existing works like LEACH and PEGASIS, the proposed EEUPA has fewer messages overhead.

Table 2. No. Of Messages Vs. Message Overhead

\begin{tabular}{|c|c|c|c|}
\hline \multirow{2}{*}{ No. of Messages } & \multicolumn{3}{|c|}{ Message Overhead (\%) } \\
\cline { 2 - 4 } & EEUPA Method & LEACH Scheme & PEGASIS \\
\hline 15 & 5 & 22 & 26 \\
\hline 30 & 11 & 29 & 32 \\
\hline 45 & 15 & 35 & 39 \\
\hline 60 & 19 & 42 & 45 \\
\hline 75 & 23 & 49 & 50 \\
\hline 90 & 28 & 53 & 59 \\
\hline 105 & 32 & 59 & 65 \\
\hline
\end{tabular}

\subsection{Network Lifespan}

The lifespan of the network is calculated depending on the number of nodes in the wireless sensor networks and the values of the proposed EEUPA is compared with the existing LEACH and PEGASIS schemes is described in Table 3.

Fig.7 explains the lifespan of the network depending on the number of nodes in the network. Because the energy drain rate of the nodes in the network environment is less, the life span of the network increases correspondingly.

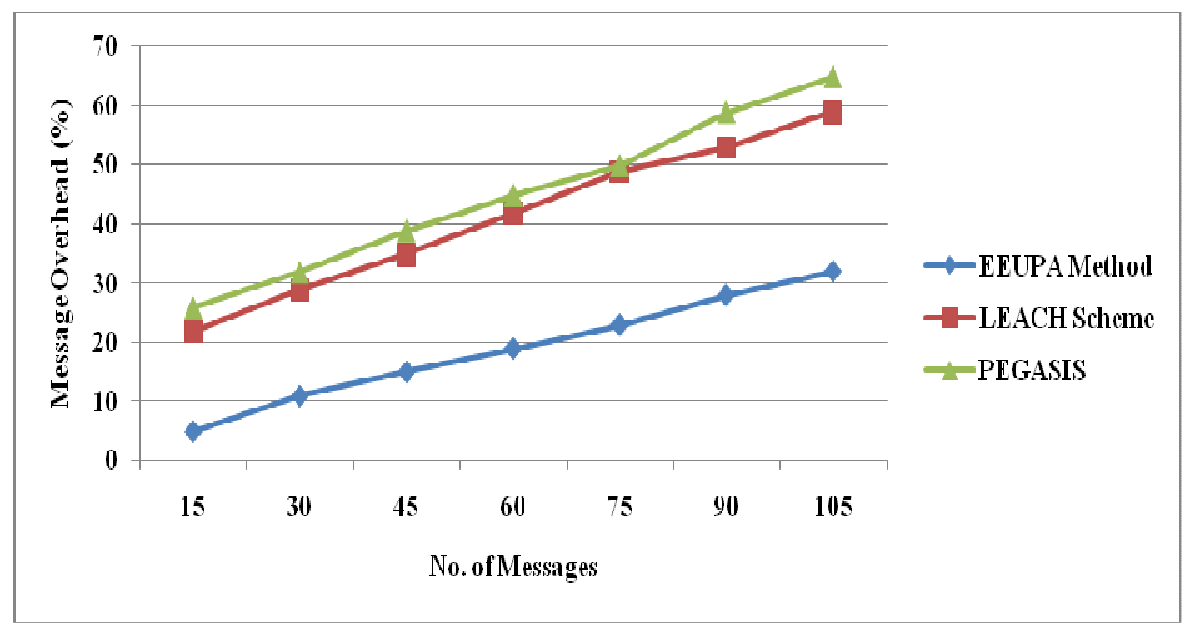

Figure 6. No. of Messages vs. Message Overhead 
International Journal on Cybernetics \& Informatics (IJCI) Vol. 4, No. 2, April 2015

Table 3. No. Of Nodes Vs. Network Lifespan

\begin{tabular}{|c|c|c|c|}
\hline \multirow{2}{*}{ No. of Nodes } & \multicolumn{3}{|c|}{ Network Lifespan (\%) } \\
\cline { 2 - 4 } & EEUPA Method & LEACH Scheme & PEGASIS \\
\hline 25 & 85 & 65 & 45 \\
\hline 50 & 87 & 68 & 49 \\
\hline 75 & 89 & 70 & 52 \\
\hline 100 & 90 & 74 & 56 \\
\hline 125 & 93 & 77 & 60 \\
\hline 150 & 95 & 80 & 64 \\
\hline 175 & 96 & 83 & 69 \\
\hline 200 & 98 & 85 & 73 \\
\hline
\end{tabular}

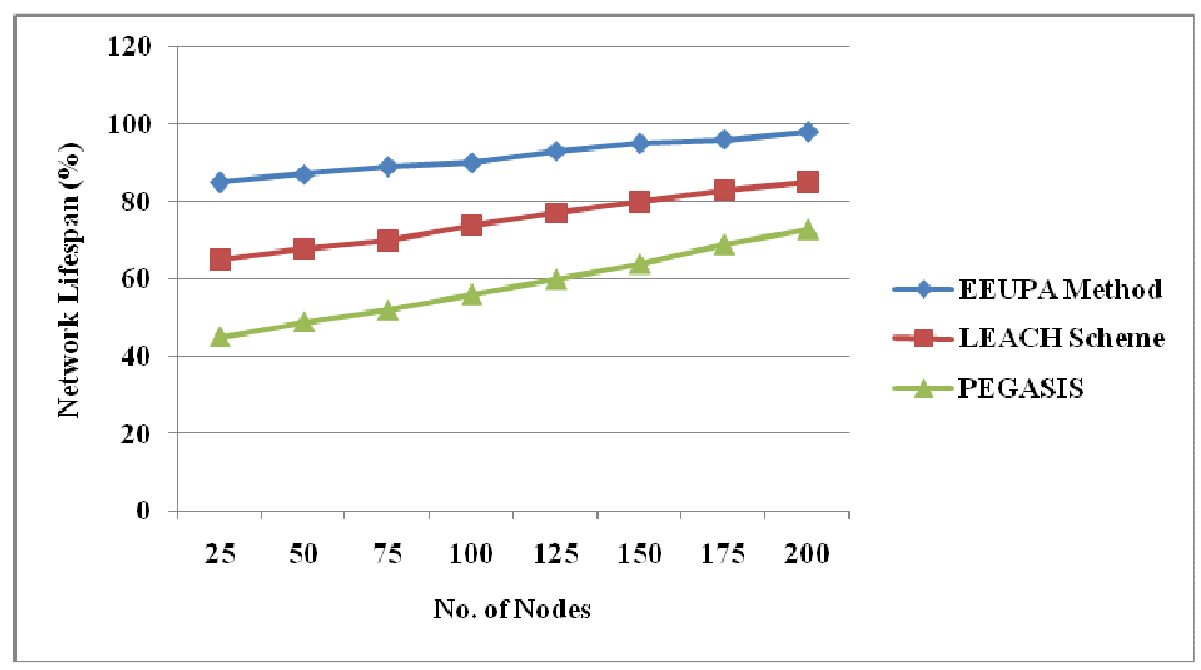

Figure 7. No. of Nodes vs. Network Lifespan

Compared to the existing works like LEACH and PEGASIS, the proposed EEUPA work attains high network lifetime as the utilization of minimal energy drain rate nodes on data collection gene series increases the lifespan of the node and the network.

\section{CONCLUSION}

In this paper we proposed a new method which is employed to calculate the lifespan of nodes constant with present traffic conditions. We described a mechanism, called the EEUPA which cannot be utilized in any of the present wireless sensor path protocol as a way organization principle. This metric is good at reflecting the current dissipation of energy without reflecting on other traffic measurements, like queue length and the number of links passing via the nodes. The main objective of EEUPA is not only to increase the lifespan of every node, but also to extend the lifespan of every link by means of selecting the consistent path from source to 
International Journal on Cybernetics \& Informatics (IJCI) Vol. 4, No. 2, April 2015

destination. With NS-2 simulator, the EEUPA method is compared with the existing works like LEACH and PEGASIS, and the results explained that the EEUPA evades over dissipation of energy, since the sequence path for the particular node is processed with allele of gene traits.

\section{REFERENCES}

[1] Chien-Ming Chen., Yue-Hsun Lin., Ya-Ching Lin., and Hung-Min Sun., "RCDA: Recoverable Concealed Data Aggregation for Data Integrity in Wireless Sensor Networks," IEEE Transactions on parallel and distributed systems Vol. 23, no. 4, 2012.

[2] Hongjuan Li., Kai Lin., Keqiu Li., "Energy-efficient and high-accuracy secure data aggregation in wireless sensor networks," Journal on Computer Communications 2011.

[3] Leandro A. Villas., Azzedine Boukerche, Daniel L. Guidoni., Horacio A.B.F. de Oliveira.,Regina Borges de Araujo., Antonio A.F. Loureiro., "An energy-aware spatio-temporal correlation mechanism to perform efficient data collection in wireless sensor networks," Journal on Computer Communications 2012.

[4] Sudip Misra., P. Dias Thomasinous., "A simple, least-time, and energy-efficient routing protocol with one-level data aggregation for wireless sensor networks," The Journal of Systems and Software, 2010.

[5] Haitao Zhang., Huadong Maa., Xiang-Yang Li., Shaojie Tang., Xiaohua Xu., "Energy-efficient scheduling with delay constraints for wireless sensor networks: A calculus-based perspective," Journal on Computer Communications 2012.

[6] Jang-Ping Sheu., Prasan Kumar Sahoo.,Chang-Hsin Su., Wei-Kai Huc., " Efficient path planning and data gathering protocols for the wireless sensor network," Journal on Computer Communications 2010.

[7] Kai Lin a., Min Chenb., Sherali Zeadally., Joel J.P.C. Rodrigues., "Balancing energy consumption with mobile agents in wireless sensor networks," Future Generation Computer Systems 2012.

[8] Massimo Vecchio., Aline Carneiro Viana., Artur Ziviani., Roy Friedman., "DEEP: Density-based proactive data dissemination protocol for wireless sensor networks with uncontrolled sink mobility," Journal on Computer Communications 2010.

[9] Basma M. Mohammad El-Basioni., Sherine M. Abd El-kader., Hussein S. Eissa.,Mohammed M. Zahra., "An Optimized Energy-aware Routing Protocol for Wireless Sensor Network," Egyptian Informatics Journal, 2011.

[10] Chung-Horng Lung., Chenjuan Zhou., "Using hierarchical agglomerative clustering in wireless sensor networks: An energy-efficient and flexible approach,” Ad Hoc Networks 2010.

[11] Manish Kumar Jhaa., Atul Kumar Pandeyb., Dipankar Pala., Anand Mohanc., ” An energy-efficient multi-layer MAC (ML-MAC) protocol for wireless sensor networks," International Journal of Electronics and Communications (AEÜ), 2011.

[12] Jun Yuea., Weiming Zhang., Weidong Xiao., Daquan Tang., Jiuyang Tang., "Energy Efficient and Balanced Cluster-Based Data Aggregation Algorithm for Wireless Sensor Networks," International Workshop on Information and Electronics Engineering (IWIEE), 2012.

[13] Muhammad Saleem., Israr Ullah., Muddassar Farooq., "BeeSensor: An energy-efficient and scalable routing protocol for wireless sensor networks," Journal on Information Sciences 2012.

[14] Jalel Ben-Othman., Bashir Yahya.," Energy efficient and QoS based routing protocol for wireless sensor networks," J. Parallel Distrib. Computation, 2010.

[15] Chih-Chieh Hung., Wen-Chih Peng., "Optimizing in-network aggregate queries in wireless sensor networks for energy saving,” Data \& Knowledge Engineering 2011. 